\title{
Evaluation of whole-body modalities for diagnosis of multifocal osteonecrosis-a pilot study
}

Shunichi Yokota ${ }^{1 \dagger}$, Keita Sakamoto ${ }^{2 \dagger}$, Yukie Shimizu ${ }^{2,3}$, Tsuyoshi Asano $^{1}$, Daisuke Takahashi ${ }^{1}$, Kohsuke Kudo $^{2}$, Norimasa Iwasaki $i^{1}$ and Tomohiro Shimizu ${ }^{1 *}$ (i)

\begin{abstract}
Background: This study aimed to investigate the ability of whole-body bone scintigraphy (WB-BS) in the detection of multifocal osteonecrosis (ON) compared to whole-body magnetic resonance imaging (WB-MRI) and to clarify the characteristics of patients with multifocal ON among those with ON of the femoral head (ONFH) using WB-MRI.

Methods: Forty-six patients who had symptomatic ONFH and underwent surgery in our hospital from April 2019 to October 2020 were included in the study. Data on patient demographics, including age, sex, body mass index (BMI), history of corticosteroid intake, alcohol abuse, smoking, and symptomatic joints, were collected from their medical records. All patients underwent WB-MRI and WB-BS before surgery.

Results: The agreement in the detection of ON by WB-MRI vs the uptake lesions by WB-BS in the hip joints was moderate $(k=0.584)$, while that in other joints was low $(k<0.40)$. Among the 152 joints with ON detected by WBMRI, 92 joints (60.5\%) were symptomatic, and 60 joints (39.5\%) were asymptomatic. Twelve out of the 46 (26.0\%) patients had multifocal (three or more distinct anatomical sites) ON. Nonetheless, while WB-BS detected symptomatic ON detected by WB-MRI as uptake lesions in $82.6 \%$ (76/92) of the joints, asymptomatic ON detected by WB-MRI was detected as uptake lesions in $21.7 \%(13 / 60)$ of the joints. All patients with multifocal ON had a history of steroid therapy, which was significantly higher than that in patients with oligofocal ON $(P=0.035)$. The patients with a hematologic disease had multifocal $O N$ at a higher rate $(P=0.015)$.

Conclusions: It might be difficult for WB-BS to detect the asymptomatic ON detected by WB-MRI compared to symptomatic ON. Considering the cost, examination time, and radiation exposure, WB-MRI might be useful for evaluating multifocal ON. Larger longitudinal studies evaluating the benefits of WB-MRI for detecting the risk factors for multifocal ON are required.
\end{abstract}

Keywords: Osteonecrosis, Whole-body magnetic resonance imaging, Whole-body bone scintigraphy, Steroid

\footnotetext{
* Correspondence: simitom@wg8.so-net.ne.jp

†Shunichi Yokota and Keita Sakamoto contributed equally to this work. 'Department of Orthopaedic Surgery, Faculty of Medicine and Graduate School of Medicine, Hokkaido University, Kita-15 Nishi-7, Kita-ku, Sapporo 060-8638, Japan

Full list of author information is available at the end of the article
}

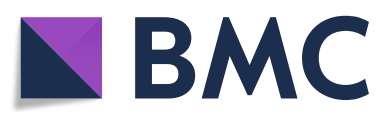

(c) The Author(s). 2021 Open Access This article is licensed under a Creative Commons Attribution 4.0 International License, which permits use, sharing, adaptation, distribution and reproduction in any medium or format, as long as you give appropriate credit to the original author(s) and the source, provide a link to the Creative Commons licence, and indicate if changes were made. The images or other third party material in this article are included in the article's Creative Commons licence, unless indicated otherwise in a credit line to the material. If material is not included in the article's Creative Commons licence and your intended use is not permitted by statutory regulation or exceeds the permitted use, you will need to obtain permission directly from the copyright holder. To view a copy of this licence, visit http://creativecommons.org/licenses/by/4.0/ The Creative Commons Public Domain Dedication waiver (http://creativecommons.org/publicdomain/zero/1.0/) applies to the data made available in this article, unless otherwise stated in a credit line to the data. 


\section{Background}

Osteonecrosis $(\mathrm{ON})$ is characterized by the death of osteocytes due to inadequate blood supply caused by various underlying mechanisms [1]. Although various joints can be affected, including the shoulders, knees, and ankles, ON of the femoral head (ONFH) is most common, with an annual incidence rate of 10,000 to 20 , 000 new cases in the USA and 2000 to 3000 new cases in Japan, which is expected to increase continuously [2-4]. Multifocal $\mathrm{ON}$ is a rare disorder involving three or more distinct anatomical sites and occurs in approximately $3 \%$ of all ON patients [5].

Imaging is used to diagnose $\mathrm{ON}$ and evaluate the severity of lesions. Radiographs are often used initially, but the early-stage disease is usually undetectable because the radiographic abnormalities develop only after prolonged ischemic changes $[6,7]$. A whole-body bone scan (WB-BS) is more sensitive than a simple X-ray scan for the diagnosis of $\mathrm{ON}$ [8-10] and can also be used to screen for multifocal $\mathrm{ON}[7,11]$. However, some reports have suggested that the sensitivity of WB-BS for the diagnosis of symptomatic ON is low $[12,13]$. Therefore, the advantages of WB-BS for screening multifocal ON remain debatable.

Magnetic resonance imaging (MRI), on the other hand, is the gold standard for the diagnosis of ON and evaluation of its severity, with high specificity and sensitivity $[14,15]$. Conventional MRI includes limited scans and does not cover all joints because of the high cost [7]. Whole-body (WB) MRI (WB-MRI) has emerged in clinical use for the management of inflammatory diseases such as rheumatoid arthritis, spondylarthritis, polymyositis, and dermatomyositis [16, 17]. It also helps in the early detection of osteonecrotic lesions in patients with a hematologic disease treated with corticosteroids $[18,19]$. Considering the high accuracy of MRI in the early identification of ON, WB-MRI allows all potential sites of $\mathrm{ON}$ to be evaluated in a single examination, thereby improving the prognosis.

However, there is little evidence supporting its utility in the detection of multifocal ON. Moreover, no studies have compared WB-MRI and WB-BS for the detection of ON. Therefore, this study aimed to investigate the ability of WB-BS in the detection of multifocal ON compared to WB-MRI and to clarify the characteristics of patients with multifocal ON among those with ONFH using WB-MRI.

\section{Methods}

\section{Patients}

This cross-sectional study was conducted in accordance with the ethical standards of the Declaration of Helsinki and approved by our institutional review board (\# 0200059). All patients were informed about this study and agreed to its publication. In total, 46 patients who had symptomatic ONFH and underwent surgery in our hospital from April 2019 to October 2020 were eligible to participate in this study. All patients were diagnosed of ONFH by three orthopedic surgeons (TA, DT, and TS) according to the diagnostic criteria reported by Sugano et al. [20]. Additionally, all patients underwent WB-MRI and WB-BS preoperatively.

Data on patient demographics, including age, sex, body mass index (BMI), history of corticosteroid intake, alcohol abuse, and smoking, were collected from their medical records. Alcohol abuse was defined as the consumption of more than $400 \mathrm{~mL}$ of alcohol per week, which is known to be a significant risk factor for ONFH [21].

\section{Whole-body MRI}

WB-MRI was performed using a 1.5-T MRI system (Magnetom Avanto; Siemens Healthcare, Erlangen, Germany) (Fig. 1a). T1-weighted (T1W) turbo spinecho images (TR 600-800 ms, TE $14 \mathrm{~ms}$, slice thickness $6 \mathrm{~mm}$, slice gap $12 \mathrm{~mm}$ in the axial section and with 3 $\mathrm{mm}$ in the coronal section) were obtained from the neck to the ankles (Fig. 1b). The total scan time was 20-25 min. While ON is asymptomatic before the collapse and is characterized by a band-like pattern in the T1-weighted images, it becomes symptomatic and painful after the collapse because of bone marrow edema and joint effusion. Therefore, in this study, T1-weighted images were selected because they help in evaluating asymptomatic ON. All MRIs were performed during the hospitalization.

\section{Whole-body bone scintigraphy}

WB-BS was performed $4 \mathrm{~h}$ after injection of $555 \mathrm{MBq}$ technetium-99 $\mathrm{m}$ hydroxymethylene diphosphonate (Tc$99 \mathrm{~m} \mathrm{HDP})$. Anterior and posterior views were acquired using a dual-headed gamma camera system (ECAM, Siemens Healthcare, Erlangen, Germany). Images were analyzed on a Siemens ESOFT Workstation.

\section{Image interpretation and analysis}

Two board-certified radiologists with 12 years of experience (K.S. and Y.S.), blind to the clinical information, evaluated the WB-MRI and WB-BS images. ON was defined as either a subchondral or an intramedullary area demarcated by a distinct marginal rim with a low signal intensity that encompassed the medullary fat on the MRI images. For evaluation of WB-BS, bone regions exhibiting uptake were recorded as previously described [7].

\section{Statistical analysis}

To compare the characteristics of cases with multifocal vs oligofocal $\mathrm{ON}$, continuous and categorical variables were analyzed by independent Student's $t$-test and 

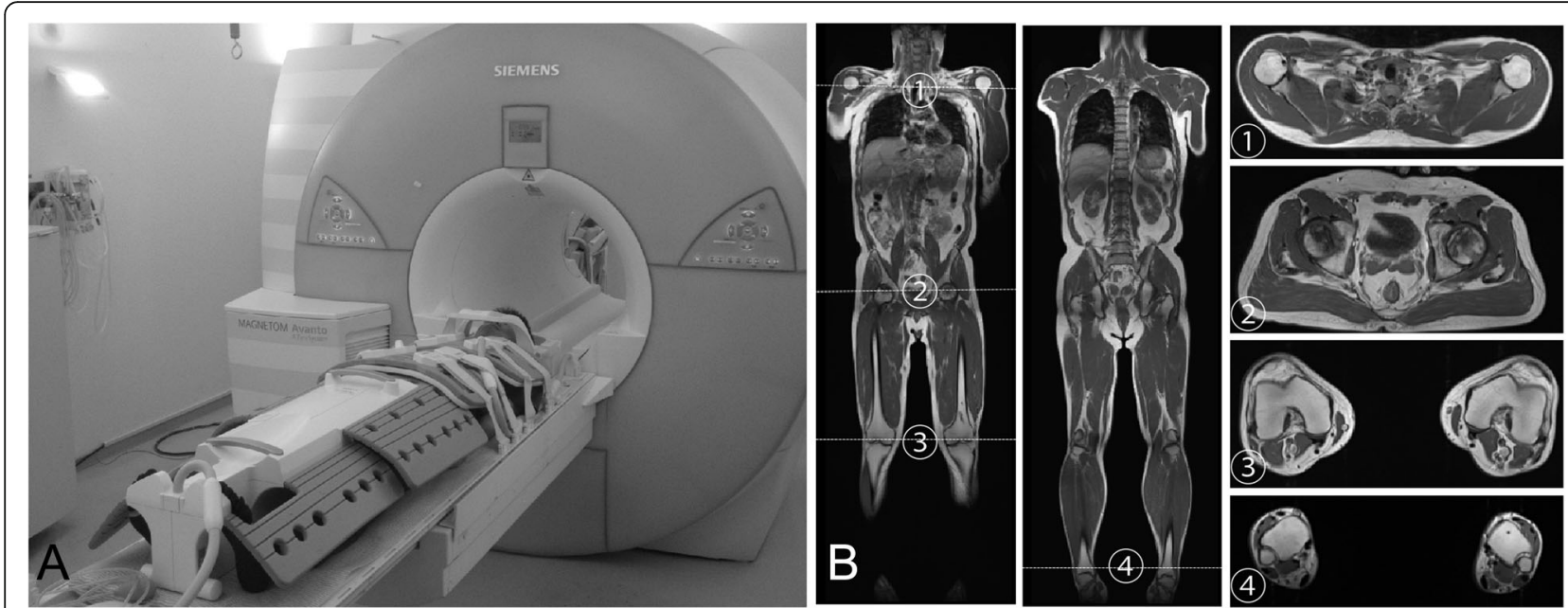

Fig. 1 Whole-body MRI. a Set-up of coils (head, chest, abdomen, and extremity coils) in a wide bore magnet device. b Typical coronal and axial images using T1-weighted (T1W) turbo spin-echo images of whole-body MRI. (1) Shoulder joints, (2) hip joints, (3) knee joints, and (4) ankle joints. MRI, magnetic resonance imaging

Pearson's chi-square test, respectively. $P$-values $<0.05$ were considered significant. Cohen kappa coefficient of the agreement was calculated to evaluate the reproducibility and accuracy of the analysis.

\section{Results}

The demographics and clinical data of the patients are summarized in Table 1 . In total, 36 out of the 46 patients received steroid therapy such as prednisolone. Of these 36 patients, 20 had collagen disease, including systemic lupus erythematosus (SLE), dermatomyositis (DM), and polymyositis (PM); 6 had hematologic malignancies; 4 had allergies; 2 had pneumonia; and 4 patients had other diseases. Fifteen out of the 36 patients received steroid pulse therapy, with a mean maximum

Table 1 Patient demographics

\begin{tabular}{ll}
\hline 46 patients & $47.1(16.0)$ \\
\hline Age, years & $23: 23$ \\
Sex, male to female & 36 \\
Steroid use, cases & \\
Comorbidity for steroid use, cases & 21 \\
$\quad$ Collagen disease & 6 \\
Hematologic malignancy & 4 \\
Allergy & 2 \\
Pneumonia & 4 \\
Others & 15 \\
Steroid pulse therapy, cases & 50.8 (17.5) \\
Mean maximum steroid dose, mg & 19 \\
Alcohol abuse, cases & 28 \\
Smoking, cases &
\end{tabular}

Data are represented as the mean (standard deviation) dose of $50.8 \mathrm{mg}$ (9-90 mg) per day. Nineteen and $28 \mathrm{pa}-$ tients had a history of alcohol abuse and smoking, respectively.

WB-MRI detected osteonecrotic lesions in 82 hip joints of 46 patients, 40 knee joints of 24 patients, 17 ankle joints of 11 patients, 9 shoulder joints of 5 patients, and 4 elbow joints of 3 patients (Table 2). Among the 152 joints evaluated, WB-MRI detected symptomatic and asymptomatic ON in $92(60.5 \%)$ and 60 (39.5\%) joints, respectively (Table 3). Twelve out of the 46 patients had multifocal ON detected by MRI. Table 4 compares the demographics of patients with multifocal and oligofocal ON, detected by WB-MRI. All patients with multifocal ON had a history of steroid therapy. Although these patients had a higher ratio of steroid use than those with oligofocal $\mathrm{ON}(P=0.035)$, there were no significant differences in the history of steroid pulse therapy and maximum dose of steroid use between the two groups. The patients with hematologic disease had multifocal $\mathrm{ON}$ at a higher rate $(P=0.015)$. There were also no significant differences in age, sex, alcohol abuse, and smoking status between the two groups.

However, WB-BS detected uptake lesions in 71 hip joints of 46 patients, 18 knee joints of 16 patients, 21 ankle joints of 13 patients, 30 shoulder joints of 16 patients, and 7 elbow joints of 5 patients (Table 2). While the agreement of the detection of $\mathrm{ON}$ in the hip joints was moderate and comparable between WB-MRI and WB-BS, that in the knee, ankle, shoulder, and elbow joints showed lower agreement (Table 2) (Fig. 2). WBBS detected symptomatic uptake lesion in 76/147 (51.7\%) joints (Table 3). Although WB-BS detected symptomatic ON detected by WB-MRI as uptake lesions in $82.6 \%(76 / 92)$ of the joints, asymptomatic $\mathrm{ON}$ 
Table 2 Number of cases of ON detected by WB-MRI and uptake lesions by WB-BS

\begin{tabular}{|c|c|c|c|c|c|}
\hline & ON detect & & Uptake les & & \\
\hline & Patient \# & Joint \# & Patient \# & Joint \# & $\kappa$ statistic \\
\hline Hip joint & 46 & 82 & 46 & 71 & 0.584 \\
\hline Knee joint & 24 & 40 & 16 & 18 & 0.069 \\
\hline Ankle joint & 11 & 17 & 13 & 21 & 0.135 \\
\hline Shoulder joint & 5 & 9 & 16 & 30 & 0.244 \\
\hline Elbow joint & 3 & 4 & 5 & 7 & 0.067 \\
\hline Total & & 152 & & 147 & \\
\hline Multifocal cases & 12 & & 14 & & \\
\hline
\end{tabular}

WB-MRI whole-body magnetic resonance imaging, WB-BS whole-body bone scintigraphy, ON osteonecrosis, "\#" number

detected by WB-MRI was detected as uptake lesions in $21.7 \%(13 / 60)$ of the joints.

The interobserver variabilities in the detection of $\mathrm{ON}$ by WB-MRI and WB-BS between two observers (K.S. and Y.S.) were 0.742 (good) and 0.554 (moderate), respectively. When they had a different diagnosis on the same patients, the radiologists and orthopedic surgeon (TS) discussed and consented if the region was adopted as an occurrence of ON or not.

\section{Discussion}

To clarify if WB-BS uptake lesions are useful for diagnosing the multifocal $\mathrm{ON}$, this study aimed to compare the detection of ON by WB-MRI and WB-BS. The main limitation of this study was that we did not perform a bone biopsy for definitive diagnosis other than femoral head received THA; therefore, this study could not evaluate the sensitivity and specificity of each modality. However, considering that previous studies reported that MRI is the gold standard for the diagnosis of $\mathrm{ON}$ and evaluation of its severity, with high specificity and sensitivity $[14,15]$, we believe that WB-MRI is an attractive and accurate tool to screen the multifocal ON. In this current study, we found that WB-BS could detect symptomatic $\mathrm{ON}$ in the lower limbs in more than $80 \%$ of the detected cases by WB-MRI, while it was not as effective in detecting asymptomatic $\mathrm{ON}$ in the upper limbs. These findings suggest that WB-BS is less efficient in detecting pre-collapse in joints other than the hip joint, where precollapse ON has a "cold-in-hot" appearance [20]. Although a previous study showed that pinhole bone scan of the shoulder, hip, knee, and ankle could provide better diagnostic values [22], WB-BS might not be enough resolution for screening multifocal ON, including asymptomatic ON. Recent studies have shown the effectiveness of regenerative therapy using growth factor or cell for ON before a potential collapse [23, 24]. Therefore, early diagnosis of ON might be important in asymptomatic patients. Additionally, considering the examination time (WB-MRI, 20-30 min, and WB-BS, $4 \mathrm{~h}$ after injection), cost (WBMRI, 200 dollars, and WB-BS, 500 dollars, in Japan), and the radiation exposure, indication of WB-BS for screening ON should be considered carefully.

This current study also investigated the clinical characteristics of patients with multiple $\mathrm{ON}$. The incidence of multifocal $\mathrm{ON}$ in this study $(26.0 \%)$ was higher than that reported previously $[5,7]$. This discrepancy could be due to the sensitivity of WB-MRI, allowing detection of asymptomatic ON. WB-MRI has been recently reported to detect multifocal $\mathrm{ON}$ in $20 \%$ (3 of 15) of the patients with polymyositis/dermatomyositis (PM/DM) [19] and in 86\% (6 of 7) of the patients with Hodgkin's lymphoma treated by chemotherapy [25]. Consistent with these reports, this current study showed that the

Table 3 Number of symptomatic and asymptomatic patients with radiological abnormality

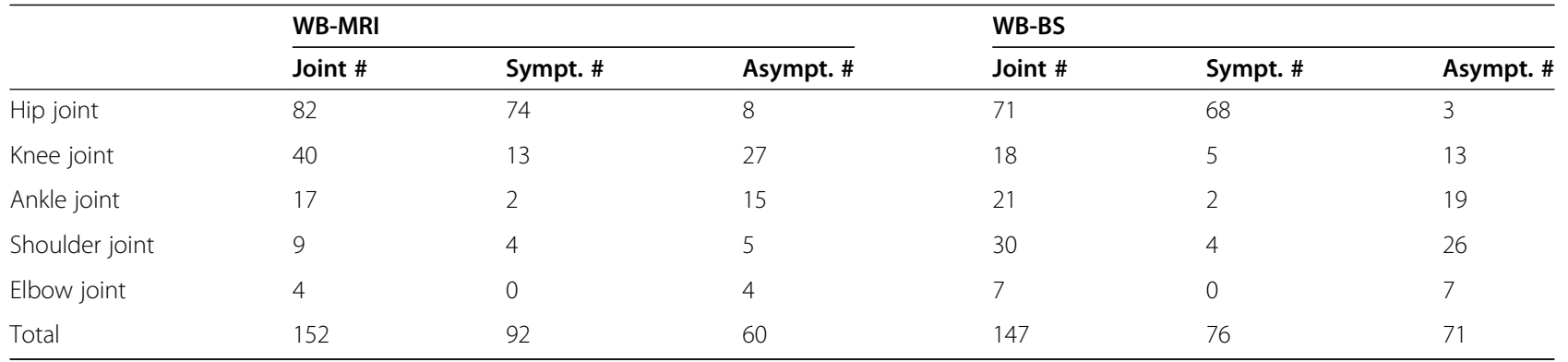

WB-MRI whole-body magnetic resonance imaging, WB-BS whole-body bone scintigraphy, Sympt symptomatic, Asympt asymptomatic, "\#" number 
Table 4 Comparison of demographics between patients with multifocal and oligofocal ON

\begin{tabular}{llll}
\hline & Multifocal ON $(\boldsymbol{N}=\mathbf{1 2})$ & Oligofocal ON $(\boldsymbol{N}=\mathbf{3 4})$ & $\boldsymbol{P}$-value \\
\hline Age, years & $42.6(12.2)$ & $48.7(16.9)$ & 0.875 \\
Sex, male to female & $4: 8$ & $15: 19$ & 0.179 \\
Steroid use, cases & 12 & 24 & 0.034 \\
Steroid pulse therapy, cases & 6 & 9 & 0.135 \\
Mean maximum steroid dose, mg & $58.0(20.1)$ & $47.5(15.8)$ & 0.198 \\
Collagen disease, cases & 6 & 15 & 0.725 \\
Hematologic disease, cases & 4 & 2 & 0.015 \\
Alcohol abuse, cases & 5 & 14 & 0.976 \\
Smoking, cases & 6 & 22 & 0.370 \\
\hline
\end{tabular}

Data are represented as the mean (standard deviation)

ON osteonecrosis

ONFH patients with hematologic disease had multifocal ON with high frequency. The incidence of multifocal $\mathrm{ON}$ is high in the presence of clinical risk factors, such as connective tissue disorders including SLE, renal transplantation, leukemia, HIV, coagulation disorders, and sickle cell disease [7, 26, 27]. Because this current study included patients with various comorbidities and symptomatic ONFH, future studies should investigate the incidence of multifocal $\mathrm{ON}$ among the patients with each comorbidity.

Consistent with the previous reports [28], this current study showed that multifocal ON occurs in steroid associated cases of ONFH. Contrary to a report by Zhang et al. [29], we found no significant difference in the maximum steroid dose for steroid pulse therapy between patients with multifocal and oligofocal ON. This discrepancy could be because Zhang et al. studied patients with severe acute respiratory syndrome [29]. Therefore, future studies should also investigate the association between the incidence of multifocal $\mathrm{ON}$ and the associated risk factors such as steroid usage under the condition of unified cases.

The present study had several limitations. First is the small sample size, which included patients with multifocal $\mathrm{ON}$ and various other comorbidities. Larger and longitudinal cohort studies using WB-MRI should be conducted to detect the risk factors for multifocal ON. Second, this study lacked bone biopsy and pathological

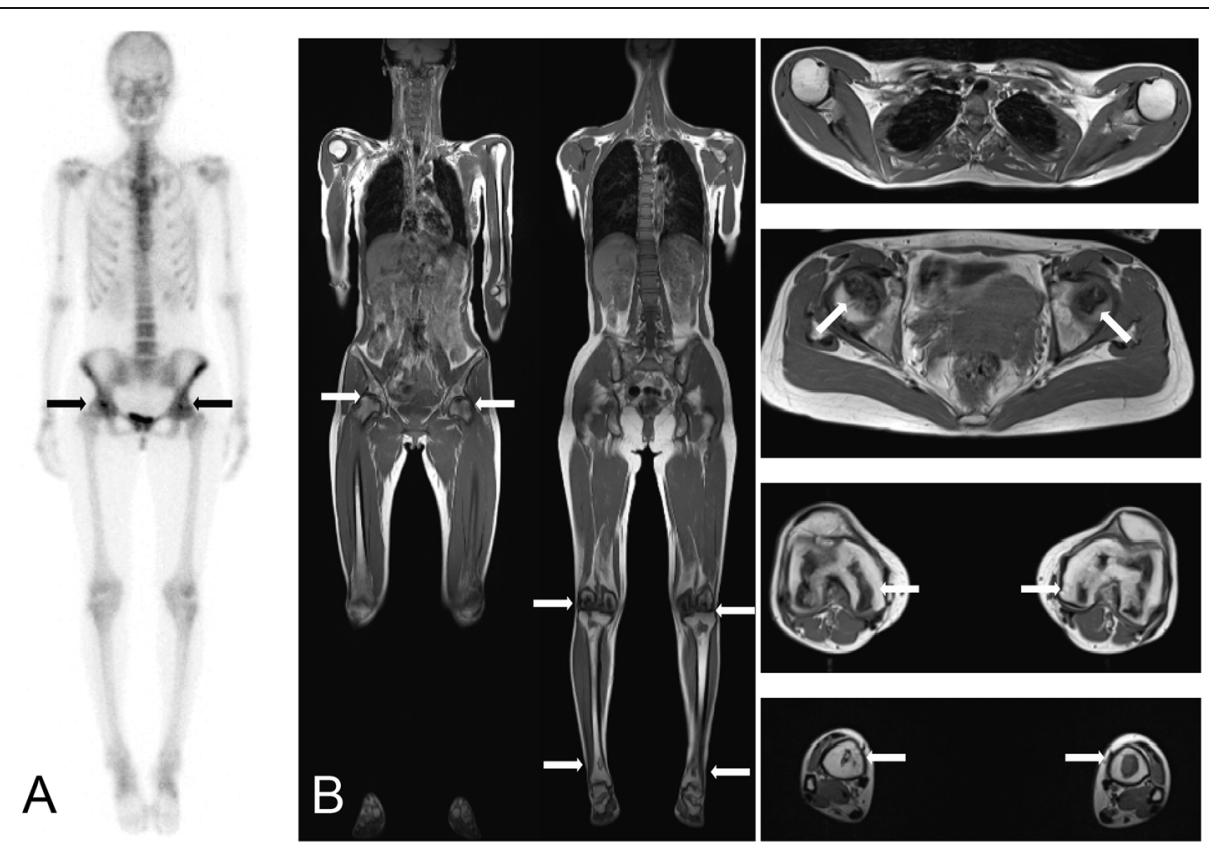

Fig. 2 Osteonecrosis of the bilateral hip, knee, and ankle joints in a 38-year-old woman with a history of steroid use for scleroderma. a Coronal whole-body bone scintigraphy image. The arrows indicate the region of uptake in the bilateral hip joints. b Coronal and axial whole-body magnetic resonance image. The arrows indicate osteonecrotic regions in the bilateral hip, knee, and ankle joints 
data. There is a possibility that other diseases such as subchondral insufficiency fracture or rapidly destructive coxarthrosis were misdiagnosed as ON. Third, the study has been conducted only on patients with symptomatic ONFH admitted to the hospital for surgical procedures. Hence, there is a selection bias as well.

\section{Conclusions}

In conclusion, it might be difficult for WB-BS to detect the asymptomatic ON detected by WB-MRI compared to symptomatic ON. Considering the cost, examination time, and radiation exposure, WB-MRI might be useful for evaluating multifocal ON. Larger longitudinal studies evaluating the benefits of WB-MRI for detecting the risk factors for multifocal $\mathrm{ON}$ are required.

\section{Abbreviations}

BMI: Body mass index; MRI: Magnetic resonance imaging; ON: Osteonecrosis; ONFH: Osteonecrosis of femoral head; T1W: T1-weighted; WB-BS: Wholebody bone scintigraphy; WB-MRI: Whole-body magnetic resonance imaging

\section{Acknowledgements}

We would like to thank Editage (www.editage.com) for English language editing.

\section{Authors' contributions}

SY and KS equally contributed to the design of the study, analysis and interpretation of the results, and writing the manuscript. YS contributed to analysis and interpretation of the data. TA contributed to the design of the study and interpretation of the data. DT, KK, and NI contributed to the design of the study and final proofreading. TS contributed to the design of the study, analysis and interpretation of the result, writing the manuscript, and final proofreading. Approval of the final version of the manuscript was obtained from all coauthors.

\section{Funding}

The study was supported by a Grant-in-Aid for Young Scientists from the Ministry of Education, Culture, Sports, Science, and Technology of Japan (20K17984).

\section{Availability of data and materials}

The datasets used and/or analyzed during the present study are available from the corresponding author on reasonable request.

\section{Declarations}

\section{Ethics approval and consent to participate}

The Institutional Review Board for Human Research approved this study design (\#020-0059).

\section{Consent for publication}

Not applicable.

\section{Competing interests}

The authors declare no competing interests.

\section{Author details}

'Department of Orthopaedic Surgery, Faculty of Medicine and Graduate School of Medicine, Hokkaido University, Kita-15 Nishi-7, Kita-ku, Sapporo 060-8638, Japan. ${ }^{2}$ Department of Diagnostic Imaging, Faculty of Medicine and Graduate School of Medicine, Hokkaido University, Kita-15 Nishi-7, Kita-ku, Sapporo 060-8638, Japan. ${ }^{3}$ Department of advanced diagnostic imaging development, Faculty of Medicine and Graduate School of Medicine, Hokkaido University, Kita-15 Nishi-7, Kita-ku, Sapporo 060-8638, Japan.
Received: 20 November 2020 Accepted: 4 March 2021

Published online: 11 March 2021

\section{References}

1. Matthews AH, Davis DD, Fish MJ, Stitson D: Avascular Necrosis. In: StatPearls. Treasure Island (FL); 2021.

2. Jacobs B. Epidemiology of traumatic and nontraumatic osteonecrosis. Clin Orthop Relat Res. 1978;130:51-67.

3. Mont MA, Hungerford DS. Non-traumatic avascular necrosis of the femoral head. J Bone Joint Surg Am. 1995;77(3):459-74.

4. Fukushima W, Fujioka M, Kubo T, Tamakoshi A, Nagai M, Hirota Y. Nationwide epidemiologic survey of idiopathic osteonecrosis of the femoral head. Clin Orthop Relat Res. 2010;468(10):2715-24.

5. LaPorte DM, Mont MA, Mohan V, Jones LC, Hungerford DS. Multifocal osteonecrosis. J Rheumatol. 1998:25(10):1968-74.

6. Grigoriu V, Stefaniu A, Fica N. Evolution of a bronchial foreign body (a tooth chip). Rev Chir Oncol Radiol O R L Oftalmol Stomatol Otorinolaringol. 1985; 30(4):311-4.

7. An YS, Park S, Jung JY, Suh $\mathrm{CH}$, Kim HA. Clinical characteristics and role of whole-body bone scan in multifocal osteonecrosis. BMC Musculoskelet Disord. 2019;20(1):23.

8. Alavi A, McCloskey JR, Steinberg ME. Early detction of avascular necrosis of the femoral head by $99 \mathrm{~m}$ technetium diphosphonate bone scan: a preliminary report. Clin Orthop Relat Res. 1977;127:137-41.

9. Gregg PJ, Walder DN. Scintigraphy versus radiography in the early diagnosis of experimental bone necrosis, with special reference to caisson disease of bone. J Bone Joint Surg Br. 1980;62-B(2):214-21.

10. Conklin JJ, Alderson PO, Zizic TM, Hungerford DS, Densereaux JY, Gober A Wagner HN. Comparison of bone scan and radiograph sensitivity in the detection of steroid-induced ischemic necrosis of bone. Radiology. 1983; 147(1):221-6.

11. Minoves M, Riera E, Costansa JM, Bassa P, Setoain J, Domenech FM. Multiple aseptic bone necrosis detected by Tc-99m MDP bone scintigraphy in a patient with systemic lupus erythematosus on corticosteroid therapy. Clin Nucl Med. 1998;23(1):48-9.

12. Assouline-Dayan Y, Chang C, Greenspan A, Shoenfeld Y, Gershwin ME. Pathogenesis and natural history of osteonecrosis. Semin Arthritis Rheum. 2002;32(2):94-124

13. Mont MA, Ulrich SD, Seyler TM, Smith JM, Marker DR, McGrath MS, Hungerford DS, Jones LC. Bone scanning of limited value for diagnosis of symptomatic oligofocal and multifocal osteonecrosis. J Rheumatol. 2008; 35(8):1629-34.

14. Fordyce MJ, Solomon L. Early detection of avascular necrosis of the femoral head by MRI. J Bone Joint Surg Br. 1993;75(3):365-7.

15. Petrigliano FA, Lieberman JR. Osteonecrosis of the hip: novel approaches to evaluation and treatment. Clin Orthop Relat Res. 2007;465:53-62.

16. O'Connell MJ, Powell T, Brennan D, Lynch T, McCarthy CJ, Eustace SJ. Whole-body MR imaging in the diagnosis of polymyositis. AJR Am J Roentgenol. 2002;179(4):967-71.

17. Cantwell C, Ryan M, O'Connell M, Cunningham P, Brennan D, Costigan D, Lynch T, Eustace S. A comparison of inflammatory myopathies at wholebody turbo STIR MRI. Clin Radiol. 2005;60(2):261-7.

18. Albano D, Patti C, Lagalla R, Midiri M, Galia M. Whole-body MRI, FDG-PET/CT, and bone marrow biopsy, for the assessment of bone marrow involvement in patients with newly diagnosed lymphoma. J Magn Reson Imaging. 2017; 45(4):1082-9.

19. Zhen-Guo H, Min-Xing Y, Xiao-Liang C, Ran Y, He C, Bao-Xiang G, Li-Guo Y, Kai-Ning S, Chan Q, Guo-Chun W. Value of whole-body magnetic resonance imaging for screening multifocal osteonecrosis in patients with polymyositis/dermatomyositis. Br J Radiol. 2017;90(1073):20160780.

20. Sugano N, Kubo T, Takaoka K, Ohzono K, Hotokebuchi T, Matsumoto T, Igarashi H, Ninomiya S. Diagnostic criteria for non-traumatic osteonecrosis of the femoral head. A multicentre study. J Bone Joint Surg Br. 1999;81(4): 590-5.

21. Matsuo K, Hirohata T, Sugioka Y, Ikeda M, Fukuda A. Influence of alcohol intake, cigarette smoking, and occupational status on idiopathic osteonecrosis of the femoral head. Clin Orthop Relat Res. 1988;234:115-23.

22. Sakai T, Sugano N, Nishii T, Haraguchi K, Yoshikawa H, Ohzono K. Bone scintigraphy for osteonecrosis of the knee in patients with non-traumatic osteonecrosis of the femoral head: comparison with magnetic resonance imaging. Ann Rheum Dis. 2001;60(1):14-20. 
23. Kuroda Y, Asada R, So K, Yonezawa A, Nankaku M, Mukai K, Ito-Ihara T, Tada $\mathrm{H}$, Yamamoto M, Murayama T, et al. A pilot study of regenerative therapy using controlled release of recombinant human fibroblast growth factor for patients with pre-collapse osteonecrosis of the femoral head. Int Orthop. 2016;40(8):1747-54.

24. Tomaru Y, Yoshioka T, Sugaya H, Aoto K, Wada H, Akaogi H, Yamazaki M, Mishima $\mathrm{H}$. Hip preserving surgery with concentrated autologous bone marrow aspirate transplantation for the treatment of asymptomatic osteonecrosis of the femoral head: retrospective review of clinical and radiological outcomes at 6 years postoperatively. BMC Musculoskelet Disord. 2017:18(1):292

25. Albano D, Patti C, La Grutta L, Grassedonio E, Mule A, Brancatelli G, Lagalla R, Midiri M, Galia M. Osteonecrosis detected by whole body magnetic resonance in patients with Hodgkin lymphoma treated by BEACOPP. Eur Radiol. 2017:27(5):2129-36.

26. Gonzalez Garcia A, Sifuentes Giraldo WA, Blazquez Canamero MA, Ahijon Lana M, Navas Elorza E, Vazquez DM. Multifocal osteonecrosis associated with human immunodeficiency virus infection. Reumatol Clin. 2012;8(6): $361-4$.

27. Sun W, Shi Z, Gao F, Wang B, Li Z. The pathogenesis of multifocal osteonecrosis. Sci Rep. 2016;6:29576.

28. El Gamal TA, El-Bakoury A, Hawkins A, Ed AlTayeb Mussa M, Er Ahmed Sweed T, Eh Samir Ansara S. Bilateral osteonecrosis of the femoral and humeral heads after short term corticosteroid therapy. A case study. Ortop Traumatol Rehabil. 2016;18(2):187-90.

29. Zhang NF, Li ZR, Wei HY, Liu ZH, Hernigou P. Steroid-induced osteonecrosis: the number of lesions is related to the dosage. J Bone Joint Surg Br. 2008; 90(9):1239-43.

\section{Publisher's Note}

Springer Nature remains neutral with regard to jurisdictional claims in published maps and institutional affiliations.

Ready to submit your research? Choose BMC and benefit from:

- fast, convenient online submission

- thorough peer review by experienced researchers in your field

- rapid publication on acceptance

- support for research data, including large and complex data types

- gold Open Access which fosters wider collaboration and increased citations

- maximum visibility for your research: over $100 \mathrm{M}$ website views per year

At $\mathrm{BMC}$, research is always in progress.

Learn more biomedcentral.com/submissions 